\title{
Physics at HERA and Beyond
}

\author{
Max Klein \\ DESY, 15738 Zeuthen, Platanenallee 6 \\ klein@ifh.de
}

\begin{abstract}
About two years prior to the planned termination of the operation of HERA, a short summary is given of the status of HERA, the physics goals of data taking until 2007, of the proposed experimental programme termed HERA III, and of ideas as to how deep inelastic scattering may conquer the TeV scale of energy.
\end{abstract}

Keywords: QCD, DIS, proton structure, electron-proton scattering, strong interactions

PACS: 12.38.-t,29.20.Dh

\section{INTRODUCTION}

This talk was given in the session devoted to the future of deep inelastic scattering (DIS). In 2001, the DESY directorate announced that HERA operation would be terminated in 2006 since one had promised to give PETRA to the synchrotron light physics community for building a $3^{\text {rd }}$ generation brilliant light source and because DESY had hoped to have begun already with the construction of TESLA at Hamburg. Thus since the end of 2001, with a workshop promptly held at Durham, the HERA community had to think about its future, and, more generally, about the future of deep inelastic scattering. While this had been a more and more pressing question, which is not resolved today, most of the efforts naturally went into the realisation of the luminosity upgrade of HERA, the challenge caused by the modified interaction regions (IR) of H1 and ZEUS and the excitement real data and results cause.

In the years $1992-2000$ the collider experiments took about $110 \mathrm{pb}^{-1}$ of positron proton data and nearly $20 \mathrm{pb}^{-1}$ with electrons. The mean specific luminosity of the HERA I phase was about $0.4 \cdot 10^{29} \mathrm{~cm}^{-2} \mathrm{~s}^{-1} / \mathrm{mA}^{2}$. The $e^{ \pm}$beams were unpolarised for H1 and ZEUS, and to about 50-60\% longitudinally polarised at HERMES. In 2001/02 the IR's were equipped with superconducting focussing quadrupoles near the vertex region thereby reducing the accessible low $Q^{2}$ range. It took time, to the fall of 2003 , until the machine in its new configuration was successfully commissioned: initially too high backgrounds were observed which in a series of systematic studies were traced back to the collisions of proton beam particles with restgas molecules steming from synchrotron radiation interaction with beam pipe and collimator elements. Modifications of the collimators at about $-1.5 \mathrm{~m}$ including additional pumps were required as was an understanding of the role and use of the cold magnet surfaces near the IR. HERA II is since then running with specific luminosities increased by a factor of $3\left(e^{+}\right)$to 5 $\left(e^{-}\right)$, lepton beam polarisations using new spin rotators around $\mathrm{H} 1$ and ZEUS of up to $50 \%$ and peak luminosity values of up to $5 \cdot 10^{31} \mathrm{~cm}^{-2} \mathrm{~s}^{-1}$, nearly as designed. At the Madison conference, May 2005, H1 and ZEUS had taken about half the HERA I $e^{+} p$ 
luminosity and about tripled the previous $e^{-} p$ luminosity values. Utilising the beam polarisation rather promptly, a long awaited, now classic DIS measurement could be done establishing the linear dependence of the charged current (CC) cross section on the lepton beam polarisation and thus finding the electroweak standard model (SM) to be succesful yet another time, see Fig.1. Many more new results obtained by the HERA collaborations were presented at this conference by ZEUS [1], H1 [2] and HERMES [3].

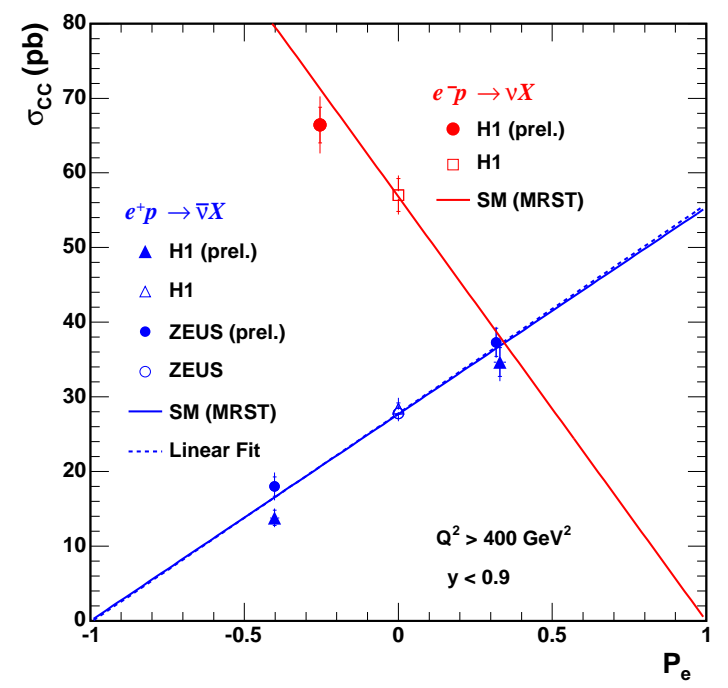

FIGURE 1. Measurements by ZEUS and $\mathrm{H} 1$ of the total cross section in deep inelastic charged current polarised electron and positron scattering as a function of the helicity of the $e^{ \pm}$beam.

This report presents a selection of physics subjects of HERA which can be expected to be investigated with enlarged statistics and higher precision than hitherto achieved. In a further section the motivation is summarised for a continued operation of HERA in a third phase. Finally ideas are presented for how deep inelastic scattering may be performed at $\mathrm{TeV}$ energies as is required for pursuing DIS physics further and for contributing to high energy physics as a whole at the new energy frontier which will be opened by the LHC.

\section{PHYSICS WITH HERA II}

Physics at HERA (II), as has been described in [4, 5, 6], is a wide field comprising classic inclusive charged and neutral current DIS, detailed tests of QCD and parton radiation, searches for new phenomena at the energy frontier and electroweak physics. So far, HERA found a number of new phenomena, in particular the rise of the proton structure function $F_{2}\left(x, Q^{2}\right)$ towards low $x$, which opened the field of high density QCD, hard diffraction which is still not explained, generalised parton distributions which lead to parton correlation measurements and exotic phenomena, specifically the observations of 5 quark states and of a few peculiar events with isolated leptons and large missing transverse momentum. It is not possible to describe this field in this contribution in any 
exhaustive way, which is why only a few possible highlights and particular challenges of physics at HERA II are briefly presented here. If the integrated luminosity gets strongly increased as is hoped for, new phenomena may become visible and rather surely new ideas will be developed in forthcoming analyses.

\section{Increase of Precision}

HERA is a laboratory for precision physics because of the very nature of deep inelastic scattering. It represents the cleanest microscope at high resolution of the world. The systematic measurement accuracy is much higher than in $p p$ colliders because the kinematics is overconstrained allowing the calorimeter energy scales to be fixed at the per cent level. The tracking and calorimetric information are combined for optimum reconstruction and the tracking and trigger efficiencies determined from the data. The luminosity is measured to $1-2 \%$ accuracy. Further improvements of the experimental accuracy are still desirable, not only because of lacking statistics at highest $Q^{2}$ and $x$, but also since most QCD effects governed by gluon radiation are logarithmic, i.e. slowly varying, and some quantities like the gluon density are determined only by derivatives of cross sections. Three major methods are being pursued to increase the accuracy: i) the luminosity is increased: at Madison one could expect HERA II to deliver about $500 \mathrm{pb}^{-1}$ of polarised $e^{ \pm}$data per experiment for analysis on tape, which would exceed the HERA I luminosity by a factor of 4 registered in about half the running time. A machine hardware improvement program had been endorsed including the exchange of all 32 "BU" magnets, large proton beam deflecting dipole magnets which exhibited aging effects, also upgrades and replacements in the proton rf., diagnostics and cryogenic systems. In order to deliver more luminosity, DESY decided to prolong the HERA life time by 6 months, until mid 2007; ii) the H1 and ZEUS detectors were upgraded substantially, in particular the forward trackers, Silicon trackers and triggers; iii) new ideas are being developed in order to cross calibrate the H1 and ZEUS measurements and thus by combining the HERA collider data significantly gaining in precision and reliability of the results [7]. Precision is the road to a success of HERA, its first and second phase.

\section{The Strong Coupling Constant}

The coupling constant $\alpha_{s}$ of the strong interaction is the worst measured fundamental coupling constant. Extrapolations of $\alpha_{e l m}, G_{F}$ and $\alpha_{s}$ to the Planck scale are uncertain, not only in principle but also due to the large error of $\alpha_{S}\left(M_{Z}^{2}\right)$ which is usually taken to be 0.003 . The determination of $\alpha_{S}\left(M_{Z}^{2}\right)$ from HERA [8] is experimentally already more precise, with a mean of $0.1186 \pm 0.0011(\exp )$ in NLO QCD. A few comments are in order here: i) the data entering this measurement are from HERA I and improvements of about a factor of two should come with higher statistics and thus enabled increased calibration accuracy and reduced systematics; ii) while this mean value seems to just agree with the world average, there has been a peculiarity which deserves final clarifi- 
cation at HERA. While the inclusion of jet data leads to a large value of $\alpha_{s}\left(M_{Z}^{2}\right)$, near 0.120 , the values from just the inclusive cross section analyses are systematically below: $\mathrm{H} 1$ data alone yield 0.115, BCDMS alone 0.110, ZEUS alone 0.110, H1 and BCDMS combined 0.115, see Fig. 2, furthermore $\mathrm{H} 1$ and NMC combined yield 0.116 [9] and the non-singlet (NNLO) analysis of [10] a value of $\alpha_{s}\left(M_{Z}^{2}\right)=0.1135$. The value of $\alpha_{s}\left(M_{Z}^{2}\right)$ is known to be particularly strongly related to the behaviour of the gluon distribution at large $x>0.3$ where inclusive DIS has little sensitivity. The contradiction, if it was confirmed at higher accuracy, may thus be due to lacking input as to how $x g$ approaches $x=1$. Yet, there may also be other reasons, as for example $\alpha_{s}\left(M_{Z}^{2}\right)$ is related to the mass of gluinos [11]; iii) to the experimental uncertainty a large, so called theoretical error is
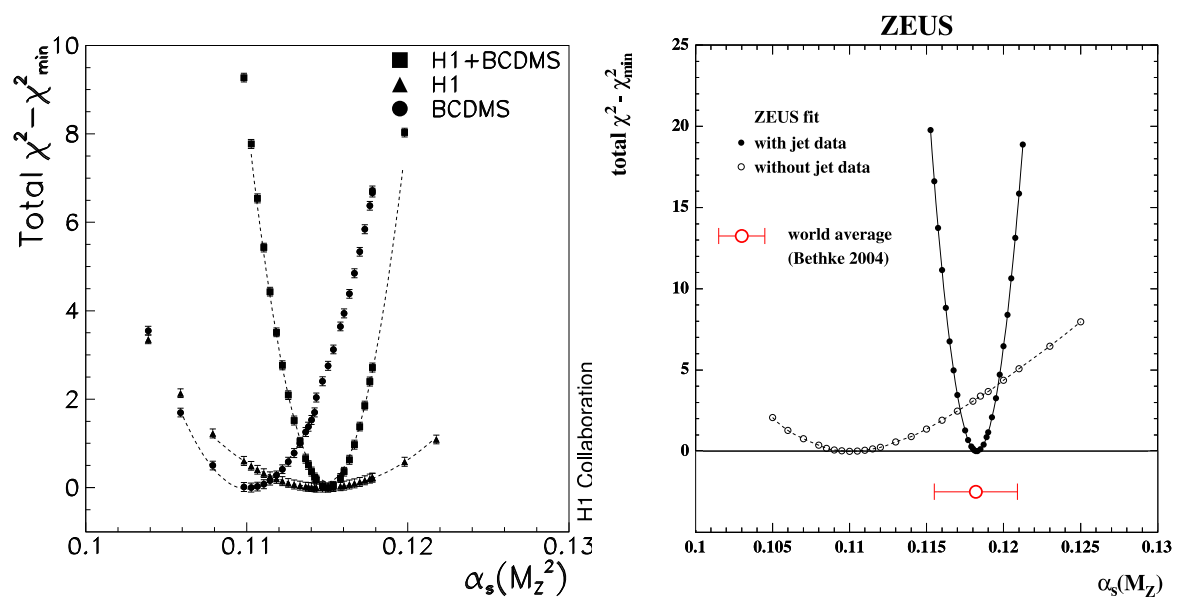

FIGURE 2. Determinations of the strong coupling constant $\alpha_{s}$ in NLO QCD fits to the BCDMS, the $\mathrm{H} 1$ and the combined $\mathrm{H} 1+\mathrm{BCDMS}$ data (left) and to the inclusive ZEUS and the combined ZEUS inclusive+jet data (right).

attached which is 0.005 in the HERA NLO analyses and stems essentially from a variation of the renormalisation (and factorisation) scale from $2 \sqrt{Q^{2}}$ to $\sqrt{Q^{2}} / 2$. This variation is an $a d h o c$ prescription the size of which is in no way supported by the inclusive DIS analyses of $\mathrm{H} 1$ and ZEUS. Therefore it deserves serious reconsideration instead of applying such variations blindly in all analyses. The calculation of NNLO splitting and coefficient functions will reduce the scale uncertainties soon, yet, the genuine influence of the scales needs to be clarified nevertheless.

\section{Heavy Flavour Physics}

Heavy flavour physics at HERA explores the charm and beauty contents of the proton and of the photon, the production and fragmentation of charmed and beauty particles. The charm contribution to the NC cross section at low $x$ is about $20 \%$ while beauty contributes to only $2 \%$ beyond threshold. Beauty physics at HERA II will play the role of charm physics at HERA I both becoming more accurately measured. At Madison first data were presented of the beauty structure function measured at low $Q^{2}$ and $x$. These and previous data at higher $Q^{2}$, both obtained with an impact parameter enrichment method based on H1's central Silicon tracker, represent the first ever measurement of $F_{2}^{b \bar{b}}$. The 
data, at the present level of accuracy, are well described by NLO QCD predictions, which determine the beauty quark density solely by the amount of scaling violations of the inclusive $F_{2}$ data and a prescription as to how beauty emerges near the threshold $Q^{2} \sim m_{b}^{2}$. Since at the LHC all heavy quark distributions are of size comparable to those of the light quarks, accurate measurements of $F_{2}^{c \bar{c}}$ and $F_{2}^{b \bar{b}}$ are important for the prediction of hard scale processes such as $W$ or $Z$ production. These distributions can directly be measured only at HERA owing to the probing character of DIS.

Interesting observations which have been made by $\mathrm{H} 1$ and ZEUS such as the excess of beauty particle production in the forward direction as compared to NLO QCD, the trend of beauty particle cross sections to exceed the NLO predictions by perhaps a factor of 1.5-2, further, the measurements of the charm fragmentation function and fractions which examine the universality of charm particle formation in $e^{+} e^{-}$versus $e p$ reactions and other measurements [1, 2], of charm jets for example, all will be performed at still increased accuracy at HERA II. This physics relies largely on the H1 and ZEUS Silicon strip detectors which have been installed as new or upgraded devices for the high luminosity phase of HERA. While the MVD of ZEUS and the CST of H1 are currently taking data, the forward and backward Silicon trackers of $\mathrm{H} 1$ are being repaired and upgraded with radiation hard electronics to be reinstalled in the shutdown 2005/06.

\section{Electroweak Physics}

HERA explores electroweak physics in the spacelike region and thus is complementary to LEP. At this workshop, the first HERA data were presented of the weak neutral current couplings of light quarks to the $Z^{0}$, see Fig. 3. These resolve the sign ambiguity
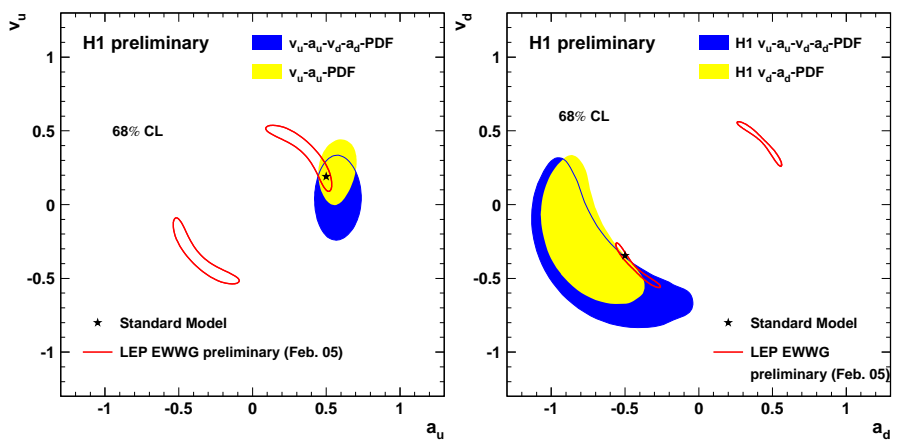

FIGURE 3. The first determinations at HERA of the weak neutral current vector and axial-vector couplings ( $v$ and $a$ ) of light quarks to the $Z_{0}$ boson compared with preliminary LEP $Z$ resonance data.

which is inherent in the LEP measurements at resonance. The result uses only the HERA I data, in particular $15 \mathrm{pb}^{-1}$ of electron data only, and represents a first important step only. Higher accuracy can be expected from the HERA II data with enlarged luminosity and lepton polarisation. At HERA parton distributions have to be known to determine electroweak parameters. One may also use electroweak interactions to probe the strong interaction: a measurement of improved precision of the $\gamma Z$ interference structure function $x G_{3}=x F_{3}^{\gamma Z}$ will allow to access the parton distributions, i.e. the valence quark com- 
bination $2 u_{V}+d_{V}$ and a possible quark-antiquark distribution difference, $q-\bar{q}$, down to $x \sim 0.002$ and determining as well the sign of the quark electric charges.

\section{Deeply Virtual Compton Scattering}

In DVCS a real photon is produced in DIS and momentum transferred between the virtual and the real photons which allows parton correlations to be accessed. Experimentally, as much as phenomenologically [17], this field is new and developing rather rapidly with the first measurements being performed at HERA [1, 2, 3] and elsewhere. DVCS interferes with Bethe-Heitler scattering and thus parton amplitudes, or generalised parton distributions, can be measured from beam charge and helicity asymmetries. In the
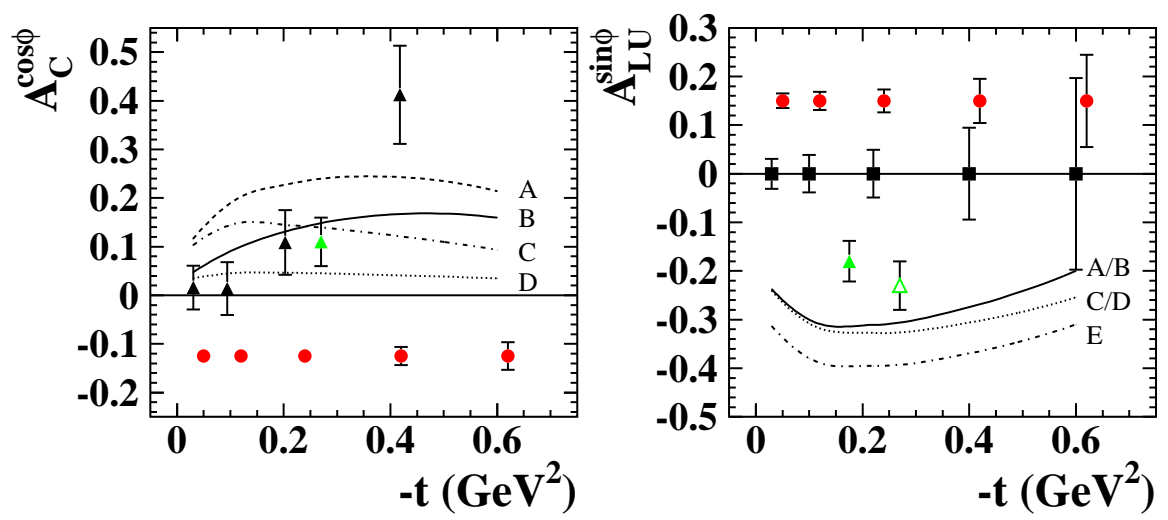

FIGURE 4. Projected accuracy for the $t$ dependence of charge (left) and beam spin asymmetries (right, assuming a polarisation of 40\%) in DVCS as expected to be measured differentially, for $x$ between 0.04 and 0.4 and $Q^{2}$ between 1 and $8 \mathrm{GeV}^{2}$, by the HERMES experiment in 2006/07 using a new fibre-Silicon detector to tag the recoiling proton. The curves are different predictions by Vanderhaegen et al.

forward limit, via the 'Ji sum rule', these may be related to the unmeasured angular momentum contribution of the quarks to the spin of the proton. HERMES intends to dedicate the final phase of HERA II to DVCS measurements, see Fig. 4, employing a newly built detector to measure the recoiling proton in elastic DVCS. New cross section and HERA II asymmetry data from H1 and ZEUS will study DVCS in a different kinematic region at low $x$.

\section{Low $x$ Physics}

HERA has established low $x$ physics, which is characterised by the dynamics of the QCD vacuum rather than primarily the structure of the proton. This field is new, the appropriate theoretical description not necessarily identical to the conventional DGLAP approach, and it is related to superhigh energy neutrino physics, to nuclear effects as studied at RHIC and ALICE and to forward physics at the LHC. Three major contribu- 
tions of HERA to this field have not been completed yet deserving further data and more unified analysis techniques. These can be sketched by the following questions:

Are the indications for a breakdown of DGLAP QCD to NLO, as observed in forward jet production, in association with dijets, and in the azimuthal decorrelation effects, true to the extent that the description of parton radiation needs to be modified in the direction of the CCFM or BFKL prescriptions? In particular which role do unintegrated parton distributions play which offer the advantage of incorporating transverse momenta, i.e the radiation kinematics, correctly?

What is the gluon distribution in the proton at low $x$ ? The gluon is determined by the $\ln Q^{2}$ derivative of $F_{2}$, which will be measured with yet increased precision. More generally, is the longitudinal structure function, which still needs to be measured at HERA, consistent with the predictions from scaling violations in QCD? The measurement of $F_{L}$ is a necessary part of the HERA II programme which tests QCD at low $x$ at higher orders. It requires a few months of data taking and highest possible precision at large inelasticities $y=Q^{2} / s x$. Additional information on $x g$ is obtained from the heavy flavour structure functions and perhaps from inelastic $J / \Psi$ production which is subject to theoretical challenges.

How should hard diffraction be integrated in low $x$ theory? If it was an absorptive correction to be subtracted prior to a $p d f$ QCD analysis [12], all distributions and their extrapolations to the LHC would be different from conventional wisdom, the desired extrapolation accuracy being $1 \%$ for determining the parton luminosity at the LHC. Moreover, the question isn't fully answered what the diffractive parton densities really are and to which extent they may be used for understanding $p p$ interactions and in particular the diffractive production of exotic states at the LHC [13]. Behind these questions are strong attempts to measure diffraction at HERA accurately, including the new Very Forward Proton Spectrometer of H1, and is the need for intimate collaboration with theory, as for most of HERA physics.

\section{Clarification of Puzzling Observations}

Much increased luminosity is required for clarification of the unexpected observations at HERA. ZEUS has reported the observation of a strange pentaquark, $\theta^{+}$, in the $K_{s}^{0} p$ channel which is not confirmed by $\mathrm{H} 1$ though the preliminary limits derived by $\mathrm{H} 1$ do not contradict the ZEUS findings. $\mathrm{H} 1$ has observed a resonance in the $D^{*} p$, the minimal quark content of which is cuudd, at an acceptance corrected rate [14] of $D^{*} p / D^{*}=1.59 \pm 0.33(\text { sta })_{-0.45}^{+0.33}($ sys $) \%$, which may be about compared with a limit of $0.35 \%$, quoted for $K \pi \pi$ and $K \pi \pi \pi D^{*}$ decays and a bit different cuts for the DIS data [15], resulting from the non-observation of such a resonance by ZEUS. These observations are being investigated with new data but still require higher statistics to be taken and the modified tracking apparatus to be carefully calibrated regarding the inclusion of Silicon detector information and material and $d E / d X$ refinements.

Since many years $\mathrm{H} 1$ is observing an excessive production of events with isolated leptons in positron proton scattering. For missing transverse momentum $p_{t}^{X}>25 \mathrm{GeV}$, and an integrated luminosity of $192 \mathrm{pb}^{-1}, 17$ events are observed and 6 expected to 
be compared with ZEUS finding 6 events at an expectation of 5.7, based on HERA I data alone. There wasn't enough electron data taken by the time of this workshop to distinguish the $e^{-} p$ from the $e^{+} p$ data, recent results, however, indicating a much less significant rate in electron scattering.

\section{HERA OPTIONS}

The future of HERA could involve an extended programme [16] including three major parts, two requiring limited luminosity while a spin collider needs very high luminosity and a proton or deuteron beam polarised:

i) The dedicated, high precision measurement of the transition from deep inelastic scattering to soft hadron collisions. The behaviour of the rise of $F_{2}$ towards low $x$ is indicative for this transition to take place for $Q^{2}$ corresponding to an inner dimension of $0.3 \mathrm{fm}$. In the proton rest frame one may view DIS as proceeding by the photon splitting into a colour dipole of variable size $2 / Q$ which interacts with the proton at an energy of $W^{2} \simeq s y=Q^{2} / x$. The distance at which the splitting occurs, the coherence length (Gribov) is large as $L \propto 1 / x$. Thus a scan of proton's structure, using both virtual photon polarisation states, would have been possible in a transition region of fundamental interest.

ii) The measurement of electron-nucleus collisions, $e d$ and $e A$. Every DIS charged lepton scattering experiment, prior to HERA, used deuterons to study the neutron structure. HERA has discovered the rise of $F_{2} \propto x(4 \bar{u}+\bar{d})$ towards low $x$. There are nonperturbative models, like the chiral quark soliton model, which predict up and down anti-quarks not to be equal. While this has been indeed confirmed at larger $x \sim 0.1$, common wisdom assumes $(\bar{d}-\bar{u}) \rightarrow 0$ at low $x$ but may yet be wrong. Deuteron data measure a singlet structure function and would allow the non-singlet and singlet evolutions at low $x$ to be separated thus contributing to the development of QCD at low $x$. The measurement accuracy of $\alpha_{S}\left(M_{Z}^{2}\right)$ would be improved by about a factor of two. An ed programme at HERA would be much more powerful than that at fixed target experiments because, by tagging the spectator proton, en scattering could be measured nearly free of Fermi motion corrections. Moreover, a diffractive programme could be pursued on $p, n$ and $d$ which also would allow the shadowing corrections to be quantified. Extending this programme to heavier nuclei would determine nuclear parton distributions, as is required for ALICE at the LHC and for RHIC. Decisive tests are possible of the predictions of a 'black body limit' of DIS, see [16], in which $F_{2} \rightarrow Q^{2} / \ln (\delta / x)$ and the diffractive scattering component reaches up to $50 \%$. Since the gluon density is amplified in nuclei as $A^{-1 / 3}$, one would exceed the unitarity limit $\sim Q^{2} / \alpha_{S}$ and be able to study saturation phenomena which in $e p$ scattering have not been observed unambiguously.

iii) With polarised protons and deuterons scattered at high luminosity off polarised leptons, HERA has the potential to make major qualitative progress in the investigation of nucleon spin components and the develoment of the QCD of polarised partons. Erraneously spin physics is sometimes considered to be nuclear physics while what it lacks is a polarised collider of high luminosity and variable beam energies such as eRHIC and HERA.

The proposed HERA III programme was widely supported and positively reviewed 
but finally ranked to be of less importance than the physics potential of the linear collider with which the programme did not intend to compete, neither in time nor financially. The result of this development is unfortunate. Building eRHIC is of interest. Not to use HERA further is wrong. The cost gained by reusing PETRA is negligible compared to the investment HERA represents. Running HERA for part time a year could have offered a way to solve manpower problems and still collect enough luminosity for the first two parts of HERA III, high precision low $x$ physics and $e N$, both requiring integrated luminosities of the order of $10 \mathrm{pb}^{-1}$ only. For the coming years, beyond 2007, DESY intends to keep the HERA proton and electron rings together which in principle offers a chance to return to HERA which still represents one of the biggest investments in particle physics.

\section{DEEP INELASTIC SCATTERING AT ENERGIES BEYOND HERA}

Particle physics is preparing for a new level of energies. Both at energies of order $10 \mathrm{GeV}$ and at the Fermi scale, explored by the colliders TeVatron, LEP and HERA, deep inelastic scattering has been making major contributions to understanding its own physics, the structure of matter and the strong interactions, and to interpreting and complementing the results from $e^{+} e^{-}$and from hadron-hadron scattering. It was a precision measurement in polarised ep scattering which in 1978 established the electron to be a right handed coupling singlet which was the final breakthrough for the $S U(2)_{L} \times U(1)$ GWS model to become standard. With HERA's $p d f$ 's, to quote another example, one is able to interpret and calculate hard scale cross sections at the TeVatron. Electroweak cross sections require LEP's precision measurements and the top quark mass prediction wouldn't have been believed without finding the top at the TeVatron. The question arises how the symbiosis of $e e, p p$ and $e p$ scattering can be ensured at the new energy scale, or specifically, how may DIS conquer the TeV scale of collision energies and why should that be a high priority task for particle physics?

\section{Towards a TeV ep Collider}

An $e p$ collider is cost effective when realised combining existing and/or future accelerators. Owing to the time structure of the cold ILC and the standing wave type cavities, it may be combined with HERA, as considered for the THERA study [19], or the TeVatron, and both arms of the linear collider may be used to realise a symmetric energy $e p$ collider of cms energy up to $2 \mathrm{TeV}$. The luminosities for THERA were estimated to range between $4 \cdot 10^{30} \mathrm{~cm}^{-2} \mathrm{~s}^{-1}$, for $E_{e}=250 \mathrm{GeV}$ and $E_{p}=1 \mathrm{TeV}$, up to $2.5 \cdot 10^{31} \mathrm{~cm}^{-2} \mathrm{~s}^{-1}$, for $E_{e}=E_{p}=500 \mathrm{GeV}$ and using dynamic focussing. A study was made [20] for a 'QCD explorer' using a CLIC prototype of $75 \mathrm{GeV}$ and the LHC proton ring of $7 \mathrm{TeV}$ with a modified, 'super bunch' structure and an estimated luminosity of $1.1 \cdot 10^{31} \mathrm{~cm}^{-2} \mathrm{~s}^{-1}$. Both THERA and the 'Explorer' would have no problems to access a much extended low $x$ region at rather large $Q^{2}$, see Fig. 5. Large rates close to the kinematic limits, however, are difficult to achieve with linac-ring combinations. 


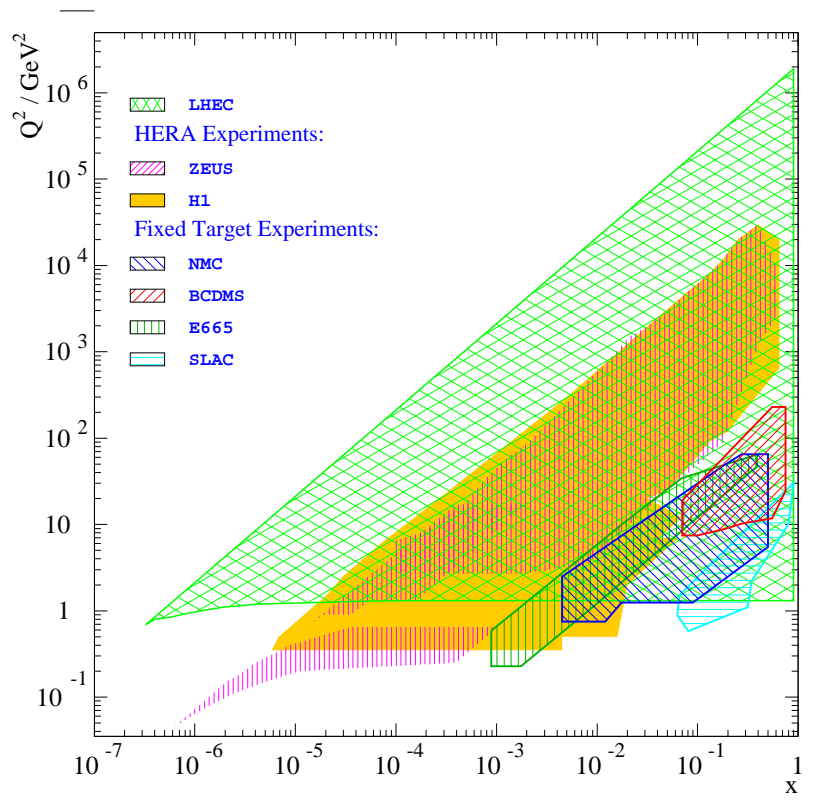

FIGURE 5. Kinematic range in Bjorken $x$ and momentum transfer squared $Q^{2}$ as covered by fixed target experiments, by $\mathrm{H} 1$ and ZEUS at HERA and by a new ep collider at the LHC at CERN or using the ILC and a $1 \mathrm{TeV}$ proton beam, from HERA at Hamburg or the TeVatron at Fermilab.

Higher luminosities in $e p$ scattering at $\mathrm{TeV}$ energies appear possible combining the LHC with an electron ring ${ }^{1}$. Early studies, first considering LEP with the LHC mounted on top [21] and then the LHC with LEP mounted on top [22], lead to estimated luminosities of $1-2 \cdot 10^{32} \mathrm{~cm}^{-2} \mathrm{~s}^{-1}$. For this workshop a tentative lattice study was made [23] of a new ep collider, LHEC, with a new electron ring mounted above the LHC. For standard LHC parameters, i.e. energy, bunch spacing, current, emittance, and a $75 \mathrm{GeV}$ electron ring of $49 \mathrm{~mA}$ current using TESLA cavities, a luminosity was estimated of $2.4 \cdot 10^{32} \mathrm{~cm}^{-2} \mathrm{~s}^{-1}$. This is achieved with strong focussing magnets near the IR limiting the acceptance range to polar angles between $10^{\circ}$ and $170^{\circ}$. Without these magnets, the luminosity is lower but the acceptance enables low $Q^{2}$ and $x$ physics to be studied. This design is being developed further regarding aspects as the luminosity, the effect of synchrotron radiation, crossing angle, the possibility of parasitic ep operations at the LHC, as well as infrastructure aspects and the feasibility to mount and close the electron ring in the LHC tunnel.

A symmetric energy machine requires an extended detector in the $e$ beam direction to measure the scattered electron at low $x$. An asymmetric machine requires special detection efforts for the forward going hadronic final state to access the region of large $x$. Detectors for an $e p$ collider at TeV energies may not pose extraordinary difficulties given the HERA experience and current technology developments.

\footnotetext{
${ }^{1}$ If the ILC was built at CERN, the possibility would appear of an ep collider of about 5 TeV energy with a maximum $Q^{2}$ of $2.8 \cdot 10^{7} \mathrm{GeV}^{2}$.
} 


\section{DIS Physics at the TeV Scale}

The physics of deep inelastic scattering at cms energies of the order of $1-2 \mathrm{TeV}$ was discussed early on [18] and much extended in a very detailed study [19], as part of the TESLA TDR. This can not be summarised here. It comprises searches for new states of matter, particles as leptoquarks, see Fig. 6, which in $e p$ can be singly produced like SUSY particles in RP violating production. Searches can be performed for substructure with highest resolution, which is the classic field of $e p$ scattering, and the partonic contents of the proton and the photon be investigated. The physics at low $x$ may qualitatively differ at the LHEC from HERA, as then the rise of the gluon density is expected to be damped by unitarity. As sketched above, there are fundamental questions which may be studied with deuterons and heavier nuclei and which are relevant for $A A$ scattering at the LHC.

For the LHC, the $p d f$ 's measured at HERA are the basis of predicting cross sections and describing the QCD processes. The extrapolations from HERA, at least at the edges of the Bjorken $x$ range, may yet be doubtful: at low $x$, in the new domain of high density QCD, gluon saturation effects possibly occuring in the LHC range may alter all HERA extrapolations, while at high $x$, the region related to the genuine, high mass discovery range at the LHC, resummation effects may similarly question such extrapolations. With new strongly interacting particles as gluinos possibly occuring in the LHC kinematic range these extrapolations would need to be altered significantly [11]. Discoveries at the LHC may as well be subtle, and high precision be required which is difficult to ensure with extrapolations over 1-2 orders of magnitude. Clearly the LHC will help defining the future of deep inelastic scattering.
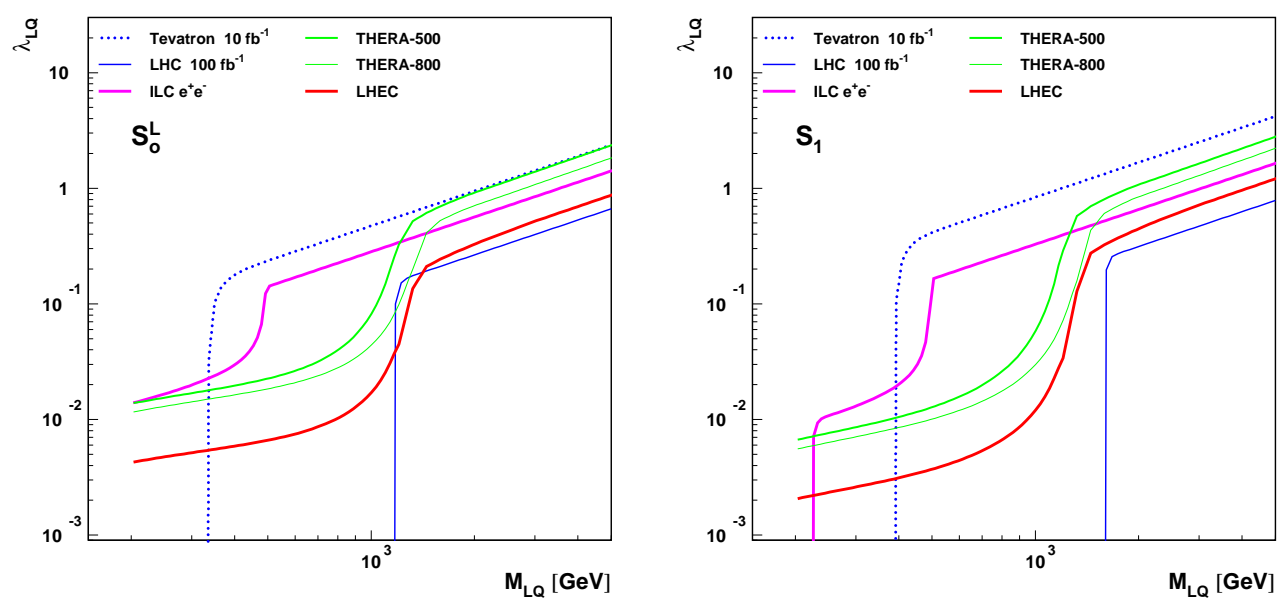

FIGURE 6. Comparison of accessible ranges for $S_{0}^{L}=e_{L} u, .$. and $S_{1}=e_{L} d, .$. leptoquark production, from [24]. As for $e p$ these particles can be singly produced and with changed initial conditions (charge and polarisation) their spectroscopy be studied, similarly for SUSY particles. Direct searches are limited by the cms energy while indirect searches may lead to higher masses. If particles are produced in the directly accessible range, the LHEC will be of much use to study particles which would be expected to be discovered at the LHC. Due to the production mechanism the next $e p$ machine has a higher range in mass than the ILC. 


\section{ACKNOWLEDGMENTS}

HERA and its physics results are due to a large community of motivated machine, experimental and theoretical physicists. I would like to thank Elke Aschenauer, Delia Hasch, Elisabetta Gallo, Rik Yoshida and many H1 colleagues for help with this talk. I thank the organisers of the session, Wesley Smith, Dave Soper and Uta Stösslein, together with Alan Caldwell, John Dainton, Tim Greenshaw, Aharon Levy, Daniel Pitzl and Ferdinand Willeke with whom and more colleagues the excitement and concern is shared for the present and the future of deep inelastic scattering.

\section{REFERENCES}

1. E. Gallo, these proceedings.

2. O. Behnke, these proceedings.

3. R. Ryckbosch, these proceedings.

4. Physics at HERA, Workshop at Ringberg, October 2003, Proceedings World Scientific, eds. G. Grindhammer, B. Kniehl and G. Kramer.

5. F. Eisele, Talk at the HERA LHC Workshop, March 2005, DESY.

6. R. Yoshida, Talk at the HERA LHC Workshop, March 2005, DESY.

7. A. Glazov, these proceedings.

8. C. Glasman, these proceedings.

9. H1 Collaboration, C. Adloff et al., Eur. Phys. J. C21,33 (2001) [hep-ex/0012053].

10. J. Blümlein, H. Böttcher and A. Guffanti, Nucl. Phys. Proc. Suppl. 135 (2004) 152 [hep-ph/0407089].

11. E. Berger et al., Phys. Rev. D71, 014007 (2005) [hep-ph/0406143].

12. A.D. Martin, M.G. Ryskin and G. Watt, Phys. Rev. D 70, 091502 (2004) [hep-ph/0406225].

13. V. Khoze, these proceedings.

14. K. Daum, these proceedings.

15. Y. Eisenberg, these proceedings.

16. T. Alexopoulos et al., eD Scattering with H1, A Letter of Intent, DESY 03-194;

H. Abramowicz et al., A New Experiment for HERA, MPP-2003-62;

F. Willeke and G. Hoffstaetter, Talks at the Workshop on the Future of DIS, Durham 2001, unpublished; http://hep.ph.liv.ac.uk/ green/HERA3/.

17. see e.g. V. Belitsky and D. Müller, Nucl. Phys. A711, 118 (2002) [hep-ph/0206306].

18. J. Feltesse and R. Rückl, Talks at the LHC Workshop, Aachen 1990, CERN 90-10 (1990), Proceedings Vol.1 p.219, 229, eds. C. Jarlskog and D. Rein.

19. The THERA Book, ep Scattering at $\sqrt{s} \sim 1$ TeV, DESY 01-123F, eds. U. Katz, M. Klein, A. Levy and S. Schlenstedt.

20. D. Schulte and F. Zimmermann, CLIC Note 608, CERN-AB-2004-079, unpublished; see also L. Gladilin et al., hep-ex/0504008.

21. A. Verdier, LHC Workshop, Aachen 1990, CERN 90-10 (1990), Proceedings Vol.2 p.890, eds. C. Jarlskog and D. Rein.

22. E. Keil, LHC Project Report 93 (1997).

23. F. Willeke, private communication.

24. A. Zarnecki, private communication. 